\title{
$\mathrm{PH} 104$ debate
}

a debate Patrimonio cultural y cambio climático

| coordina Alejandro García Hermida

\section{Seguridad cultural, Venecia y el acqua alta}

Xira Ruiz Campillo | Dpto. de Relaciones Internacionales e Historial Global, Universidad Complutense de Madrid M. a Pilar Montero Vilar | Dpto. de Pintura y Conservación-Restauración, Universidad Complutense de Madrid

URL de la contribución <www.iaph.es/revistaph/index.php/revistaph/article/view/4990>

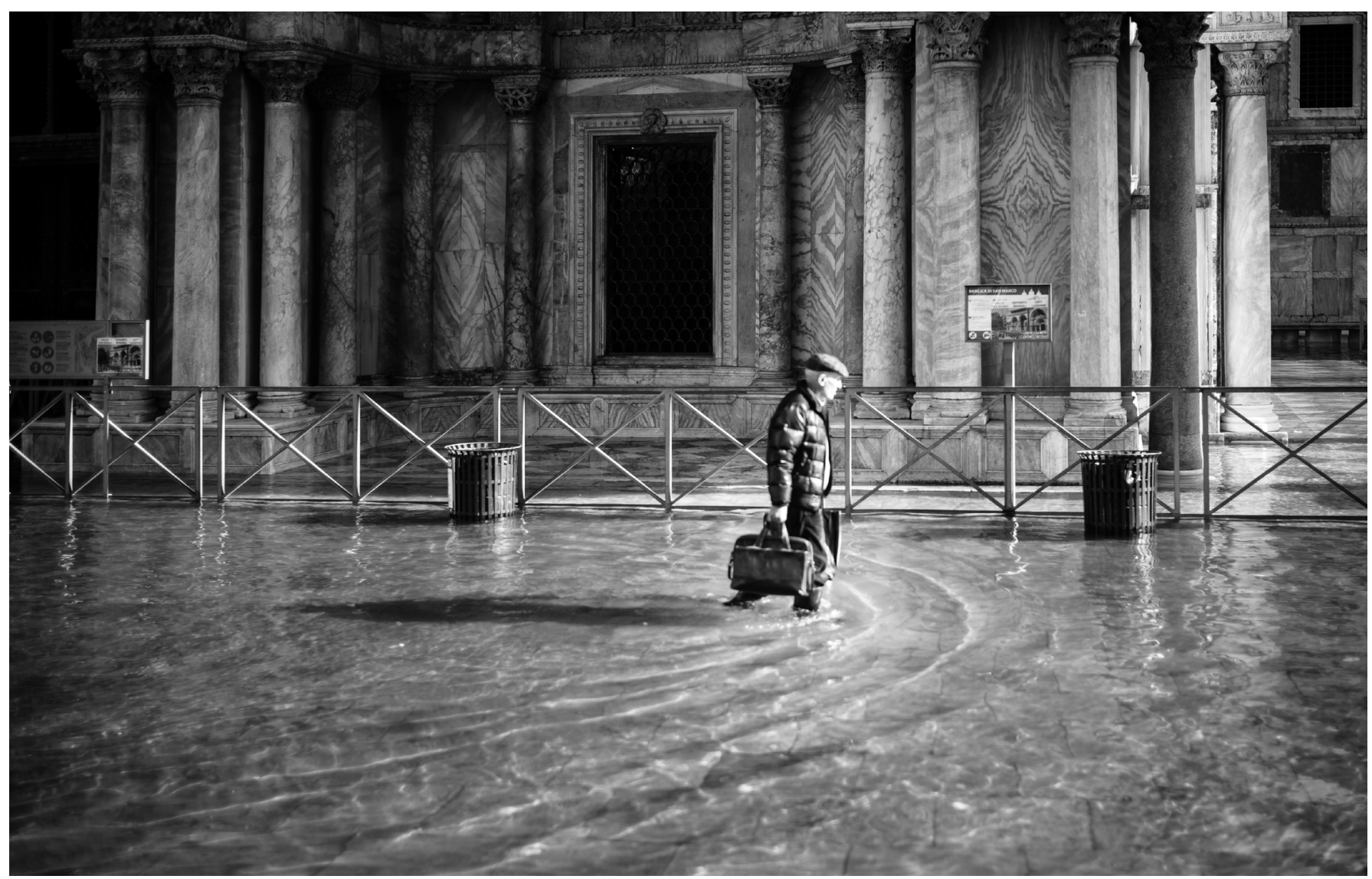

Acqua alta en Venecia, Italia | foto Tobi Gaulke

\section{Introducción}

Desde la adopción de la Convención Marco de las Naciones Unidas sobre el Cambio Climático en 1992, el conocimiento y la acción sobre este desafío global han aumentado en todo el mundo. Resulta de sobra conocido que el cambio climático está producido por la emisión de gases de efecto invernadero (GEI) procedentes de diversas actividades: desde la generación de energía hasta la producción de ropa, pasando por el reciente debate generado en España por el Ministro de Consumo sobre la relación entre la producción de carne y la emisión de GEI (Ligero y Robaina 2021). Somos conscientes de que el cambio climático impacta hasta en el más minúsculo ámbito de nuestras vidas.

Sabemos también las consecuencias de no reducir esas emisiones: el aumento de las temperaturas y su impacto 
en la falta de agua, las sequías o las olas de calor; el deshielo, los fenómenos meteorológicos extremos o la acidificación de los océanos (IPCC 2015; US Global Change Research Program 2014; Delbeke y Vis 2016; Worldwatch Institute 2009).

Sin embargo, menos se ha estudiado la relación entre el cambio climático y la cultura: ¿Hasta qué punto puede el cambio climático condicionar y cambiar nuestra cultura, nuestros valores, nuestro patrimonio histórico? ¿Por qué y cómo podemos perder nuestra seguridad cultural como consecuencia del cambio climático?

\section{Cambio climático y seguridad cultural}

El concepto de seguridad cultural ha recibido distintas definiciones en la literatura académica (Krupocin y Krupocin 2020). Algunos autores lo han relacionado especialmente con el impacto que el vandalismo y el robo, sobre todo en tiempos de guerra, puede ocasionar a la cultura de un país (Nemeth 2015). Para esta pieza de debate, vamos a tratar la seguridad cultural como una parte de la seguridad humana, concepto que se hizo muy conocido a partir de 1994 por su utilización en el Informe de Seguridad Humana del Programa de las Naciones Unidas para el Desarrollo. El concepto de seguridad humana identifica siete dimensiones en la seguridad de las personas: seguridad alimentaria, económica, salud, seguridad política, personal, medioambiental o seguridad dentro de la comunidad (UNDP 1994). Mucho se ha escrito sobre el impacto del cambio climático en cada una de ellas: en la economía (Jahn 2015), en la salud (Knowlton 2015), en la seguridad alimentaria (Crush 2012) o incluso en la seguridad personal, si relacionamos cambio climático y conflictos (Homer-Dixon 1994), pero menos sobre su impacto en la cultura.

La seguridad cultural puede contemplar desde la protección de lenguas o tradiciones y culturas ancestrales, hasta el patrimonio histórico y cultural (Krupocin y Krupocin 2020). Así, la seguridad cultural podríamos identificarla como parte de la dimensión de la seguridad de las comunidades dentro del concepto de seguridad humana. Formar parte de una comunidad nos proporciona una identidad cultural y una serie de valores y estructuras compartidas por el grupo que ofrece protección, sobre todo a los miembros más vulnerables de la sociedad. La subida del nivel del mar, los eventos meteorológicos extremos o las migraciones forzadas por la imposibilidad de continuar con la vida en la comunidad de pertenencia ponen en peligro todos estos aspectos de la seguridad cultural.

En esta pieza de debate, las autoras quieren utilizar el ejemplo de Venecia como un ejemplo vivo del impacto del cambio climático en nuestra seguridad cultural, pero también de cómo las autoridades están actuando para proteger esta ciudad tan valiosa en la cultura occidental.

\section{El acqua alta en Venecia}

La ciudad de Venecia y su laguna son patrimonio mundial de la Unesco desde 1987. La ciudad está construida sobre alrededor de cien islas, casi todas unidas entre sí por más de cuatrocientos puentes.

Su situación geográfica hace que la ciudad esté sujeta a variaciones del nivel del agua producidas por mareas y vientos que provocan que parte de la ciudad pueda verse inundada por el fenómeno conocido como acqua alta, que supone la concurrencia de varios fenómenos que en sí mismos no son generadores de desastre. Sin embargo, la simultaneidad de los mismos unidos a la vulnerabilidad del frágil equilibrio creado en el ecosistema veneciano puede llegar a provocar una pérdida irreparable.

Año tras año, entre otoño e invierno, la ciudad de los canales suele vivir en una constante disputa con el acqua alta, fenómeno que se considera cuando la subida de la marea es superior o igual a $80 \mathrm{~cm}$. Las condiciones meteorológicas que tienen que concurrir para que se produzca acqua alta en Venecia son el viento sirocco en el Adriático, que suele producirse entre octubre y enero, y ciclones extratropicales en el norte de Italia cerca de Los Alpes, que implican bajas presiones. A estas dos condiciones habría que añadirles el fenómeno astronómico de la marea alta, que también puede tener un componente 
a debate Patrimonio cultural y cambio climático

| coordina Alejandro García Hermida

meteorológico debido al estado atmosférico. Todo ello contribuye a la erosión debido a las mareas, al ataque de insectos xilófagos que consiguen alcanzar las maderas de las puertas de edificios históricos (Tagliapietra et al. 2019), o el peligro debido a la humedad que pone en riesgo murales y frescos difíciles o imposibles de trasladar y que por tanto se podrían perder. Además, hay un éxodo masivo de la ciudad, que ha pasado de 175.000 habitantes en 1951 a 51.000 en 2018, con cada vez menos gente joven y con una escasa actividad cultural propuesta centrada en la atracción de turismo y no en la retención de población (Settis 2019), lo que también contribuye a poner en peligro la seguridad cultural de la ciudad.

Desde 1872 hay registros de las veces que la ciudad se ha visto afectada por el acqua alta, observándose en 1966 la altura más alta jamás registrada, llegando el agua a $194 \mathrm{~cm}$. El aumento del nivel del mar, en crecimiento constante en los últimos 150 años (Comune de Venezia 2020), también contribuye a poner en peligro el patrimonio de la ciudad.

La laguna veneciana está altamente monitorizada como lo muestran los estudios realizados por el Instituto Superior de Investigación y Protección del Medio Ambiente (ISPRA), el Centro de Información y Previsión de Mareas del Municipio de Venecia (CPSM) y el Instituto de Ciencias Marinas de Venecia (ISMAR) del Consejo Nacional de Investigación (CNR). Los tres institutos han colaborado durante años en las actividades de vigilancia, análisis y predicción de fenómenos y de medidas del mar en la laguna de Venecia y en el Alto Adriático.

Actualmente se está estudiando cómo el cambio climático está afectando a Venecia, en la medida en que los eventos de acqua alta son más intensos en los últimos años. El año 2019 fue especialmente adverso desde el punto de vista de su frecuencia e intensidad.

El proyecto Mose(Modulo Sperimentale Elettromeccanico) comenzó a construirse en 2003 para evitar las inundaciones en los momentos de marea alta. La obra consta de 78 esclusas móviles de casi 300 toneladas y 60 metros de longitud instaladas en las bocas del puerto de la laguna: Lido norte y Lido sur, Malamocco y Chioggia. Este sistema de compuertas parece ser la única obra capaz de asegurar la protección completa del territorio contra las inundaciones, no interferir en el paisaje y no interferir en las actividades económicas de la ciudad (Ministero delle Infrastrutture e dei Trasport s.f.). El sistema mantiene las puertas de la laguna abiertas durante las mareas bajas de modo que sigue existiendo el movimiento natural del agua con la laguna. Cuando la marea sube más de 1,1 m sobre el nivel del mar, se inyecta aire en las compuertas que expulsa el agua que había en el interior, lo que bloquea la entrada de agua desde el Adriático hacia la laguna. El sistema Mose afirma poder proteger a Venecia y la laguna de mares de hasta tres metros de altura y de un aumento del nivel del mar de hasta $60 \mathrm{~cm}$ en los próximos cien años (Ministero delle Infrastrutture e dei Trasporti s.f.).

\section{Conclusión}

El impacto del cambio climático en la seguridad cultural merece mayor atención desde la literatura académica, dado que es un área aún poco explorada y desarrollada y no son pocas las consecuencias que el cambio climático puede tener en el patrimonio cultural. Aunque existen medidas de adaptación, como el sistema Mose, no están claras ni las consecuencias de este proyecto sobre el ecosistema y la biodiversidad de la laguna veneciana a medio y largo plazo, ni su funcionamiento en condiciones más adversas, ni mucho menos que este sea un sistema exportable a otros lugares con menos recursos o donde la seguridad cultural se vea amenazada por la pérdida de una lengua y costumbres.

\section{Nota}

Este trabajo forma parte del proyecto Observatorio de Emergencias y Gestión de Riesgos en Patrimonio Cultural, financiado por la convocatoria 2020 de Proyectos de Investigación Santander-Universidad Complutense de Madrid. 


\section{BIBLIOGRAFÍA}

- Comune de Venezia (2020) La subsidenza e l'eustatismo. Disponible en: https://www.comune.venezia.it/it/content/lasubsidenza-e-leustatismo [Consulta: 19/07/2021]

- Crush, J. (2012) Linking Migration, Food Security and Development. Queen (Canadá): Southern African Research Centre. Disponible en: https://media.africaportal.org/documents/ linking_migration_food_security.pdf [Consulta: 19/07/2021]

- Delbeke, J. y Vis, P. (2016) EU Climate Policy Explained. sl: European Union. Disponible en: https://ec.europa.eu/ clima/sites/clima/files/eu_climate_policy_explained_en.pdf [Consulta: 19/07/2021]

- Homer-Dixon, T. (1994) Environmental Scarcities and Violent Conflict: Evidence from Cases. International Security, vol. 19, n. ${ }^{\circ}$ 1, pp. 5-40. Disponible en: https://www.jstor.org/ stable/i323306 [Consulta: 19/07/2021]

- IPCC [Grupo Intergubernamental de Expertos sobre el Cambio Climático] (2015) Cambio climático 2014. Informe de síntesis. Disponible en: https://www.ipcc.ch/site/assets/ uploads/2018/02/SYR_AR5_FINAL_full_es.pdf [Consulta: $14 / 07 / 2021]$

- Jahn, M. (2015) Economics of extreme weather events: Terminology and regional impact models. Weather and Climate Extremes, vol. 10, part B, pp. 29-39. Disponible en: https://www. sciencedirect.com/science/article/pii/S2212094715300141 [Consulta: 19/07/2021]

- Knowlton, K. (2015) Ozone, oppressive air masses, and degraded air quality. En: Luber, G. y Lemery, J. (ed.) Global Climate Change and Human Health: from science to practice. San Francisco, CA: Jossey-Bass, a Wiley brand; Washington, DC: APHA Press, an imprint of American Public Health Association, p. 148

- Krupocin, D. y Krupocin, J. (2020) The Impact of Climate Change on Cultural Security. Journal of Strategic Security, vol. vol. 13, n. ${ }^{\circ} 4$, pp 1-28. Disponible en: https://scholarcommons. usf.edu/cgi/viewcontent.cgi ?article $=1847 \&$ context=jss [Consulta: 19/07/2021]

- Ligero, M. y Robaina, E. (2021) Comer menos carne o cuando la política engulle a la ciencia. Climática, 8 julio de 2021. Disponible en: https://www.climatica.lamarea.com/ politica-ciencia-carne-garzon/ [Consulta: 19/07/2021]

- Ministero delle Infrastrutture e dei Trasporti. Provveditorato Interregionale per le Opere Pubbliche del Veneto, Trentino Alto Adige, Friuli Venezia GiuliaMose Venezia (s.f.) Mose. Per la difesa di Venezia e della laguna dalle acque alte. Disponible en: https://www.mosevenezia.eu/ [Consulta: 19/07/2021]

- Nemeth, E. (2015) Cultural Security: Evaluating the Power of Culture in International Affairs. London: Imperial College Press
- Settis, S. (2019) Is it too late to save Venice? Apollo, 2 January 2019. Disponible en: https://www.apollo-magazine. com/is-it-too-late-to-save-venice/ [Consulta: 19/07/2021]

- Tagliapietra, D., Sigovini, M., Keppel, E., Guarneri, I., Parlanti, S., Veronese, N. y Abbate, A. (2019) Bioerosion effects of sea-level rise on the Doge's Palace water doors in Venice (Italy). Facies, 65, n. ${ }^{\circ} 34$, pp. 1-16

- United Nations Development Programme (UNDP) (1994) Human Development Report. New York; Oxford: Oxford University Press. Disponible en: http://hdr.undp.org/sites/ default/files/reports/255/hdr_1994_en_complete_nostats.pdf [Consulta: 19/07/2021]

- US Global Change Research Program (2014) The National Climate Assessment. Disponible en: https://nca2014. globalchange.gov/report [Consulta: 19/07/2021]

- Worldwatch Institute (2009) Climate Change Reference Guide. Disponible en: https://rosap.ntl.bts.gov/view/dot/17278 [Consulta: 19/07/2021] 